\title{
An electronic device for long-term snow wetness recording
}

\author{
A. DenOTH \\ Institute for Experimental Physics, University of Innsbruck, A-6020 Austria
}

\begin{abstract}
Water content, water distribution and variations with time of these quantities in the natural snow cover are important parameters for microwave remotesensing studies, snow stability investigations and snow hydrology studies. The most promising method for snow wetness determination is the measurement of the dielectric constant at radio frequencies. Recent developments of electronic devices for long-term recording of snow wetness are reported. The measurement system consists of two parts: the tuning and display unit with the battery pack and the flat capacitive sensor. The system is controlled by a built-in low-power microcomputer, the operation frequency for the sensor is $20.00 \mathrm{MHz}$. Measurement data are displayed on an LCD and are also stored in a RAM; data transfer for a PC for further data processing is possible by a built-in V24 interface. Up to four individual sensors can be operated simultaneously. Field measurements of long-term variations in snow wetness during diurnal cycles and registrations of penetrating meltwater or rain-water waves with the formation of water shock fronts are presented.
\end{abstract}

\section{INTRODUCTION}

The dielectric function of wet snow as a multi-phase heterogeneous system is, in general, controlled by the electric/dielectric properties, volume fractions of the components, the geometry and the distribution of the solid (ice) and liquid (water) constituents (Polder and van Santen, 1946; Sihvola, 1988). At measurement frequencies in the range of $10 \mathrm{MHz}$ to $\approx 1 \mathrm{GHz}$, snow permittivity is mostly controlled by snow density and wetness; it is not dependent on frequency itself. Effects of ionic conductivity or effects of size and shape of the ice grains or clusters can be neglected. The shape and type of distribution of the water component, however, shows only a small influence on snow permittivity (Denoth, 1982).

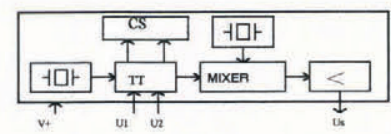

Sensor unit

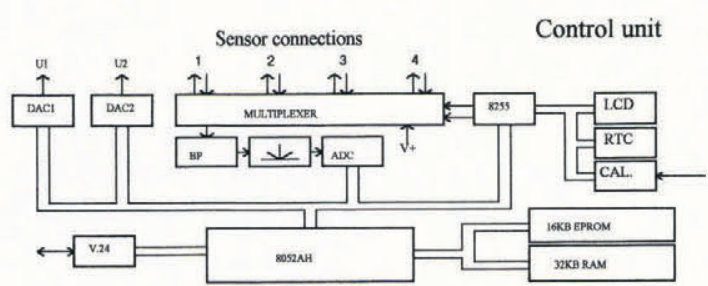

Fig. 1. Block diagram of system electronics. CS, capacitive sensor; TT, twin-T-bridge; DAC, digital-analog converter; $L C D$, Display; RTC real time clock.
Neglecting the small effects of liquid water geometry, a relatively simple relation between snow permittivity $\epsilon$, density $\rho\left(\mathrm{g} \mathrm{m}^{-3}\right)$ and volumetric water content $W$ $(\mathrm{Vol} \%)$ has been found experimentally (Denoth and others, 1984; Denoth, 1989):

$$
\epsilon=1+1.92 \rho+0.44 \rho^{2}+0.187 W+0.0045 W^{2} .
$$

Snow permittivity is measured using flat capacitive sensors to allow both near-surface and volume wetness determinations. The thickness of only $1.5 \mathrm{~mm}$ of the platelike sensor offers a nearly non-destructive measurement. For electronic simplicity, a fixed measuring frequency of $20.00 \mathrm{MHz}$ has been selected.

\section{SYSTEM ELECTRONICS}

The measuring system consists of two parts: the tuning and display unit and the sensor unit. The flat plate-like capacitive sensor is an integral part of the sensor unit, and is connected to the tuning unit by a cable. An extended block-diagram of system electronics is given in Figure 1.

The capacitive sensor (CS) is connected to a twin-Tbridge (TT) which operates at a frequency of $20.00 \mathrm{MHz}$. A diagram of the twin-T-bridge with appropriate values of the components is given in Figure 2a. Tuning of the bridge is made by a voltage-controlled variation of the capacity of two varactor diodes, D (BB105 or equivalent types). The corresponding tuning voltages, U1 and U2, are applied alternatively. Tuning is automatically 


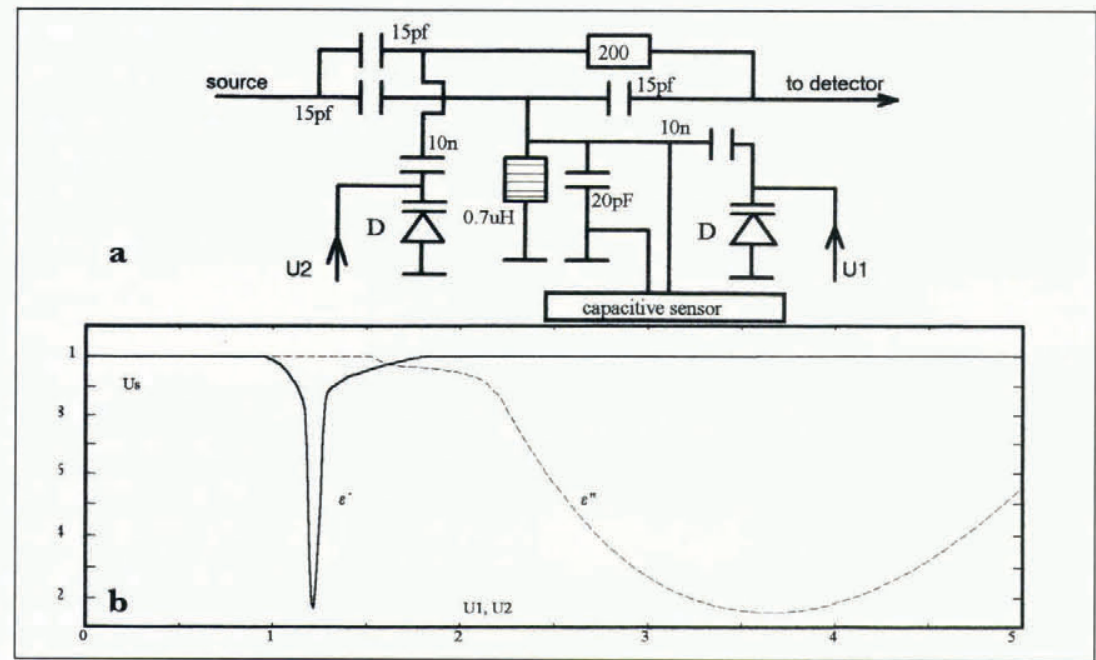

Fig. 2. a, Twin-T-bridge with appropriate values of the components; $b$, tuning characteristics of the twin-T-bridge. Us, sensor output signal; $U 1\left(\epsilon^{\prime}\right)$ and $U 2\left(\epsilon^{\prime \prime}\right)$, tuning voltages.

controlled by a low-power microcomputer, whereby the sensor output signal is downconverted to a relatively low frequency in the range of $12-14 \mathrm{kHz}$. This allows the application of relatively long cables (up to $3 \mathrm{~m}$ ) for sensor connection to the control unit. The bridge has been optimized for precise and sensitive measurements of snow permittivity, $\epsilon^{\prime}$, whereby a reduced accuracy in the determination of dielectric losses, $\epsilon^{\prime \prime}$, has been accepted. The tuning characteristics of the bridge with a sensor in air $\left(\epsilon^{\prime}=1\right)$ are illustrated in Figure 2b. The sharp minimum in the tuning voltage $\mathrm{Ul}\left(\epsilon^{\prime}\right)$ allows very accurate and sensitive measurements of $\epsilon^{\prime}$. The control unit has been designed for operation of up to four sensors simultaneously. Measured snow permittivity $\epsilon^{\prime}$ is displayed on a LCD and is also stored in a $32 \mathrm{kB}$ CMOSRAM.

A V24 interface allows data transfer to a PC for further data processing. The stored data set has a header to display the date, time and the time interval of the measurement sequence. The time interval between successive measurements and the quantity of sensors to be operated can be preselected using an external PC (programmable control unit). The minimum time interval is $20 \mathrm{~s}$ per sensor operated; the maximum is $4 \mathrm{~h}$. The system is powered by a lead accumulator with a capacity of $1.3 \mathrm{Ah}$, which allows a continuous operation of approximately $10 \mathrm{~h}$.

Calibration parameters of the capacitive sensors are determined experimentally using both solid and liquid standard dielectric materials. From two tuning operations, in air as a reference measurement $\left(U_{\text {ref }}, \epsilon^{\prime}=1\right)$, and in or on snow $(U)$ snow permittivity is calculated according to

$$
\epsilon^{\prime}=1+k \log \left(U / U_{\text {ref }}\right)
$$

whereby $k$ is the sensor-specific calibration factor. The reference measurement in air should be made with the sensor unit cooled down to snow temperature $\left(0^{\circ} \mathrm{C}\right)$. In the range from $-5^{\circ}$ to $+5^{\circ} \mathrm{C}$ sensor electronics is not sensitive to changes in ambient temperature; so the temperature coefficient of $U_{\text {ref }}$ is negligible small: $\leq-0.2 \% \mathrm{~K}^{-1} . U_{\text {ref }}$ is stored in the RAM of the control

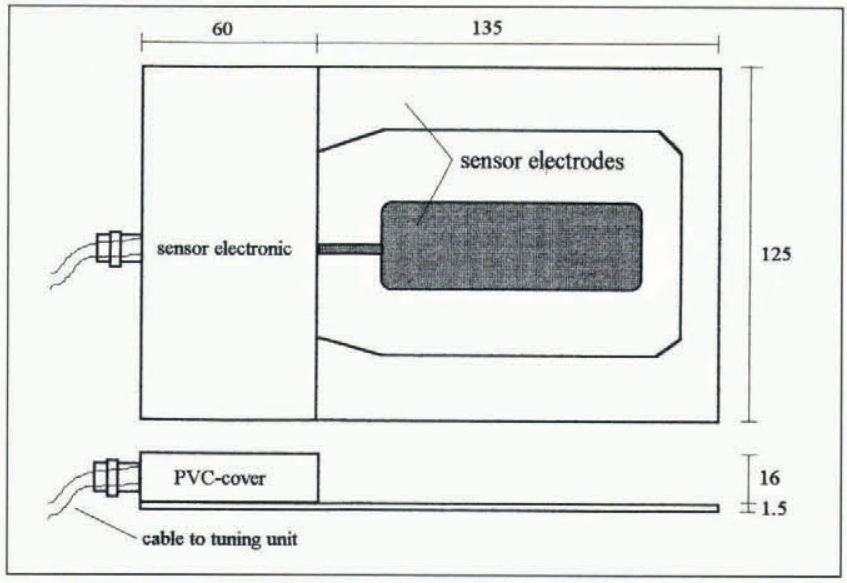

Fig. 3. The sensor unit; dimensions are given in millimeters.

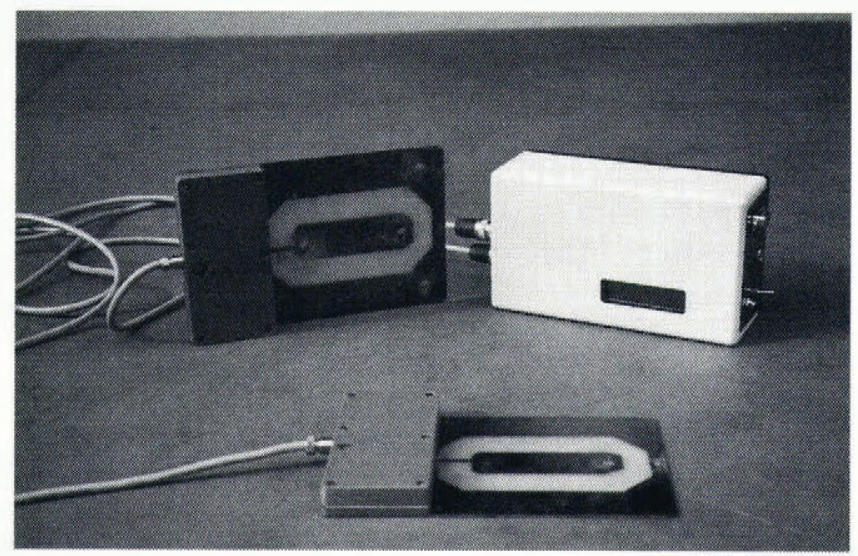

Fig. 4. Measurement system with two sensors connected to the control unit.

unit and all subsequent measurements are referred to this value. The relative error in the determination of snow permittivity is $E\left(\epsilon^{\prime}\right) \cong 2 \%$. A drawing of the sensor unit is shown in Figure 3; dimensions are given in millimeters. A photograph of the measurement system with two sensors connected to the control unit is shown in Figure 4. 


\section{FIELD MEASUREMENTS}

Typical field applications of this new computerized snow wetness meter are long-term recordings of wetness variations with simultaneously operated sensors at different depths of a snow cover and the detection of rain- or meltwater waves in snow. The sensor should be inserted vertically into the snowpack otherwise, placed

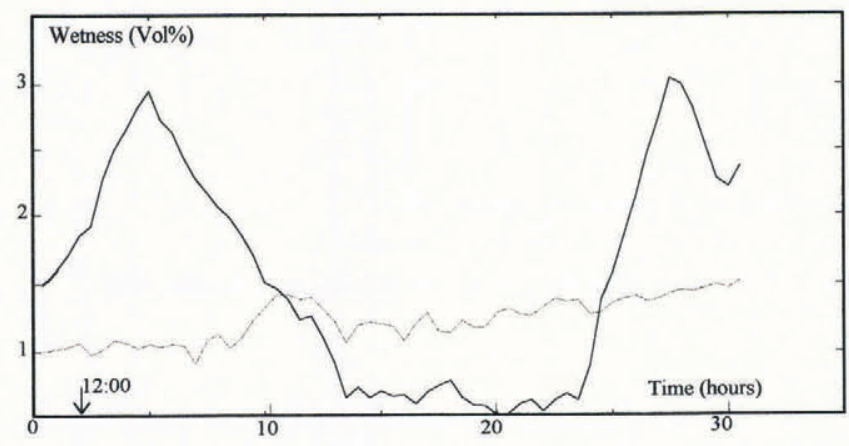

Fig. 5. Diurnal cycle in snow wetness measured at two depths of $18 \mathrm{~cm}$ (solid line) and $40 \mathrm{~cm}$ (dashed line).

horizontally, the sensor forms an impermeable layer and water percolation may be disturbed significantly. Figure 5 shows measurements of diurnal cycles in wetness variations of a natural snow cover (Seegrube, $2000 \mathrm{~m}$ a.s.l., March 1992; old snow with a mean grain-size of $\approx 0.5 \mathrm{~mm}$ ). Two sensors have been operated simultaneously at a depth of 18 and $40 \mathrm{~cm}$, respectively. The

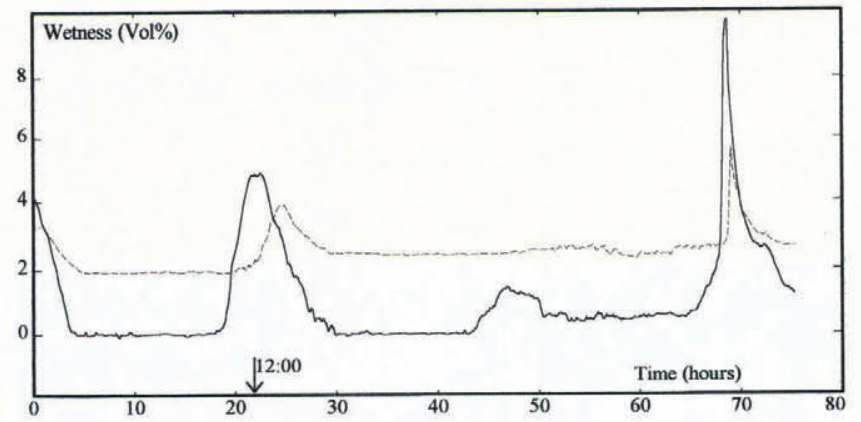

Fig. 6. Detection of water waves and water shock fronts at two depths of $10 \mathrm{~cm}$ (solid line) and $50 \mathrm{~cm}$ (dashed line) of a natural snow cover. time interval between successive measurements has been preset to $30 \mathrm{~min}$. The snow layer near the surface is characterized by relatively large and rapid changes in wetness, with a nearly constant value of $W \approx 0.6 \mathrm{Vol} \%$ overnight. Wetness at a depth of $40 \mathrm{~cm}$ shows a slow increase from $1 \mathrm{Vol}^{\%} \%$ to approximately $1.5 \mathrm{Vol} \%$ during the $30 \mathrm{~h}$ measurement sequence, whereby no marked changes have been detected. Figure 6 shows the detection of percolating water waves in a natural snow cover (Stubai Alps, $3000 \mathrm{~m}$ a.s.l., July 1991; old coarse-grained snow with a mean grain-size of $\approx 1 \mathrm{~mm}$ ). Snow wetness has been monitored near the snow surface $($ depth $10 \mathrm{~cm})$, and at a depth of $50 \mathrm{~cm}$; measurements have been made at a $10 \mathrm{~min}$ time interval. The uppermost snow layer shows marked wetness variations due to surface melting and freezing overnight. The percolating meltwater wave was recorded by the other sensor with a time delay of approximately $3 \mathrm{~h}$. A short but heavy rainfall at the third day of wetness detection caused the formation of a marked water shock front.

\section{ACKNOWLEDGEMENTS}

The Wintersport Tirol AG is thanked for supporting in part the field activities in the Stubai Alps. The Österreichische Forschungsgemeinschaft is thanked for supporting presentation through grant no. 06/2204.

\section{REFERENGES}

Denoth, A. 1982. The pendular-funicular liquid transition and snow metamorphism. 7. Glaciol., 28(99), 357-364.

Denoth, A. 1989. Snow dielectric measurements. Advances in Space Research, 9(1), 233-243.

Denoth, A. and 6 others. 1984. A comparative study of instruments for measuring the liquid water content of snow. f. Appl. Phys., 56(7), $2154-2160$

Polder, D. and J.H. van Santen. 1946. The effective permeability of mixtures of solids. Physica, 12, 257.

Sihvola, A. 1988. Self consistency aspects of dielectric mixing theory. Helsinki, Helsinki University of Technology. Faculty of Electrical Engineering. (Report 29.)

The accuracy of references in the text and in this list is the responsibility of the author, to whom queries should be addressed. 